\title{
ERRORS IN PRONOUNCING ENGLISH PHONEMES: A PRAAT ANALYSIS
}

\author{
Rohani Ganie ${ }^{1}$, Wahyu Maulana ${ }^{2}$, Rahmadsyah Rangkuti ${ }^{3}$ \\ University of Sumatera Utara (USU) \\ Medan, Indonesia \\ E-mail: ganierohani@gmail.com
}

Received: 13 May 2019

Accepted: 28 June 2019

\begin{abstract}
This article is concerned with a study of pronunciation errors made by students of English Literature Department, Faculty of Cultural Sciences, University of Sumatera Utara. The scope is limited to the study of error analysis in the pronunciation of English phonemes. The participants of this study were two students of the department with Acehnese background. In this study, the electronic software called Praat was used as an instrument in the analysis of speech sounds of the participants. The purpose of this study was to find out the dominant errors of the pronunciation of English phonemes made by the participants. The data were derived from the pronunciations of English phonemes which were recorded and transcribed in the International Phonetic Alphabet (IPA) as in Katamba (1996: 13). The standard value of the pronunciation of English phonemes was adopted from Cambridge Advanced Learner's Dictionary. The quality of the pronunciations of the participants was described in graphs and the results were shown in percentage as given in tables and charts.
\end{abstract}

Keywords: phonology, articulatory phonetics, Praat software, inter-language.

\section{Introduction}

Linguistics is the study of language systems and all their aspects: how it is structured, how it is acquired, how it is used in the production and comprehension of messages. The part of linguistics that is concerned with the structure of language is divided into a number of subfields such as: phonology (the study of speech sounds in their cognitive aspects) phonetics (the study of speech sounds in their physical aspects), morphology (the study of the formation of words), syntax (the study of the formation of sentences), semantics (the study of meaning), and pragmatics (the study of language use) (Ogden, 2009).

This study focuses on the subfield of phonetics. It is a subfield of linguistics which focuses on how speech sounds are physically produced and received. In doing a speech act, human being needs to develop a language. Language is a purely human way to communicate ideas, emotions, and desires by means of voluntarily produced symbols. Thus, speech is important to express ideas and emotions. These symbols are represented in the study of phonetics, how speech sounds are produced, what the properties of speech sounds are, and how we perceive speech.

The study of phonetics has three aspects: acoustic phonetics, auditory phonetics, and articulatory phonetics. Acoustic phonetics is the study of acoustic characteristics of speech sounds, including analysis and descriptions of speech sounds. Auditory phonetics is the study 
of physiological processes involved in the reception of speech sounds. Articulatory phonetics studies how speech sounds are produced by the organs of the vocal tract.

This study applies Praat software as it deals with acoustic aspects of speech sounds. Acoustic phonetics investigates time domain features such as the mean squared amplitude of a waveform, duration, fundamental frequency, and to abstract linguistic concepts such as phonemes, phrases or utterances. With acoustic phonetics, meaningful differences between phonemes, syllable, and words can be separated. Some aspects of speech sounds can be properly defined in acoustic terms. Therefore, the samples of this study were chosen from the students of English department of University of Sumatera Utara, who are originated from Acehnese ethnic group who have studied phonetics. The students who have passed phonetics subject are considered having enough knowledge to pronounce words in English correctly.

The purpose of this study was to find out the degree of pronunciation errors made by the participants. This study also aims at helping students understand that errors are actually the process before acquiring English like the pronunciation of the native speakers. This study was also expected to be useful to make phonology learning activities in the classroom more interesting and understandable for the students as the teachers can focus on teaching some words which are difficult to pronounce prior to those which are relatively easier so that the students can acquire the pronunciations of English phonemes perfectly.

\section{Literature Review}

The theoretical framework of this research is based on the theory of phonology proposed by Katamba (1996), particularly in the notion of phonemes and the International Phonetic Alphabets. Phonology is the branch of linguistics which investigates the ways in which sounds are used systematically in different languages to form words and utterances. Phonetics ias the study of the inventory of all speech sounds which humans are capable of producing (Katamba, 1996: 1). Phonemes refer to a family of sounds which count as the same in the language in question, which are functional (Katamba, 1996: 18). International Phonetics Alphabets (IPA) refer to the symbols conventionally used in phonology to transcribe speech sounds as in (Katamba, 1996: 13) and (Roach, 2008).

Earlier works and researches on phonology have been conducted by many linguists and researchers. Nilawati (2008) conducted a research entitled The fossilized phones errors of the English Department students of Andalas University: An Interlanguage study. She analyzes the accent of Bahasa Minang as interference to second language learning. There are 8 participants, who are English Department students, 4 with literature focus and 4 with linguistic focus. She picks the theory of Error Analysis and Inter language (Corder, 1985), and the theory of phonology (Katamba, 1989) and the theory of 'understand of fossilization' (Selinker, 1972) which becomes the references of IPA and recording. Nilawati prepares 42 words to be spoken and 15 questions for being analyzed further. This study is partially related to the present work as it is about analyzing pronunciation through an interview. Nilawati also used Praat software in her study. She held the interview with the sample using reading task. After that, the data was processed further by using Praat software. The result was then analyzed further to achieve the final result.

Another research related to the present study is published in the journal of the library of the University of Gajah Mada, Yogyakarta (i-lib, 2003) entitled Interferensi Fonologi Bahasa Indonesia dalam Bahasa Perancis 'Phonological Interference of Indonesian in French'. It explains about how the interference of mother language in second language learning. The 
result of the study shows that each language has its own uniqueness. The interference happens especially in the pronunciation of phonemes which are not found in Bahasa Indonesia such as nasal phonemes. This study focused on phonological system, syllable structure and orthographic and pronunciation system. This work is related to the present study in the case of pronunciation analysis but it does not use Praat software as its tool to analyze the data from the students' pronunciation.

The next work was conducted by Kurniawan (2016) on error analysis of the pronunciation of dental fricative consonants $(/ \theta /, / \delta /)$ by students of English education study program Faculty of Teacher Training and Education, Sriwijaya University. He randomly took 120 students from the total of 240 students of the study program to be analyzed. Some students were asked to pronounce 30 words in which some of them were distracters but all of the words were recorded and then analyzed. A native speaker was involved in analyzing the errors. The results showed that pronunciation errors occurred when they pronounced alveolar stops / $/$, and /d/. This study was really successful by involving a native speaker as the reference of standard pronunciation but the native speaker during this research was a bit different because the writer picked Google Gstatic pronunciation as the standard value of participants' pronunciation. Another difference was because this study focused only on the pronunciation of dental fricatives while the writer does a research for all English Phonemes.

\section{Research Method}

To deal with the problem, this research employs a descriptive qualitative approach. Moleong (2000) says that qualitative research often uses the quantitative data but it is generally not followed by quantitative analysis simultaneously. From the statement above, although the writer uses qualitative method, the quantitative data in numerical information is involved to describe the frequency, quantity, and intensity of the pronounced phonemes.

These steps are applied in researching the problem:

- The observation is done by downloading and recording the sound files.

- The result of the observation becomes the data which are then analyzed.

- The data are taken from native and non-native English speakers.

- The sound files are analyzed using Praat to find their acoustic features.

- Drawing the conclusion is the final point of the study.

The students majoring in English in the University of Sumatera Utara who had learned phonology were chosen as the participants of this study. Their ethnic background is Acehnese and they moved to Medan to continue their study in the university. The students also had finished their phonology class with minimum B grade and both of them were twenty years old and in the $6^{\text {th }}$ semester at the time when this research was being conducted.

In collecting the data, the writer used documentary technique through the observation. The data were collected by using a computer in the form of sound files. The writer chose recorded sounds from Cambridge Advanced Learner's Dictionary rather than from the native speaker manually because it was smoother, spending less time, energy and lower cost, and having English standard value of pronunciation.

The steps administered in collecting the data:

- The writer took the data of English native speaker's pronunciation from Cambridge Advanced Learner's Dictionary as the standard value of pronunciation. 
Errors in Pronouncing English Phonemes: A Praat Analysis, Rohani Ganie, Wahyu Maulana, Rahmadsyah Rangkuti

- The word 'example' was replaced by the words that the writer wanted to analyze, then the sound file was recorded in $\mathrm{mp} 3$ format.

- The writer recorded the pronunciation of the participants and the participants read the word containing the focused phoneme one by one.

- The format of sound files from the dictionary and participants were changed into wav for the best quality, collected into folder, and then analyzed using Praat software.

The collected data were sorted to find every part of the task and questionnaire containing the particles observed in the research. To deal with the problem, the data from the reading task were transcribed and analyzed further by using Praat software to analyze, synthesize, and manipulate sounds, and also to discover the pitch realization.

Praat is a free computer software package for speech analysis in phonetics. It was designed, and continues to be developed, by Paul Boersma and David Weenink of the University of Amsterdam. It can run on a wide range of operating systems. The program supports speech synthesis, including articulatory synthesis (https://en.wikipedia.org/wiki/ Praat).

The errors were determined by the result from Praat analysis in the form of the percentage and then the pronunciation value of the participants were compared with that of the native speaker in terms of such phonetic aspects as pitch, duration, and spectrum average value. The average value of the native speaker is considered as the standard value $(100 \%)$. This sound file of the native speaker as adopted from Cambridge Advanced Learner's Dictionary was applied as the standard value and correct pronunciation in Praat software.

\section{Results and Discussion}

The result in this study is presented in tables and graphs, mostly the pronunciation value of the participants and that of the native speaker. This part consists of / / , / /, /e/, /ə/, $/ æ /, / N /$, and $/ \mathrm{D} /$ phonemes. The result of Praat analysis is displayed in the table below.

Table 4.1 Pronounced English Short Vowels

\begin{tabular}{|c|c|c|c|c|c|}
\hline Words & $\begin{array}{l}\text { Focused } \\
\text { Phoneme }\end{array}$ & Speaker & $\begin{array}{l}\text { Frequency } \\
\text { (Pitch) }\end{array}$ & $\begin{array}{l}\text { Quantity } \\
\text { (Duration) }\end{array}$ & $\begin{array}{l}\text { Intensity } \\
\text { (Spectrum) }\end{array}$ \\
\hline \multirow{3}{*}{ Fish } & \multirow{3}{*}{ /1/ } & NS & 199.3055784 & 0.1176190 & 80.6099988 \\
\hline & & P1 & 248.3028882 & 0.1164399 & 68.8030532 \\
\hline & & P2 & 249.7549003 & 0.1360544 & 75.8113738 \\
\hline \multirow{3}{*}{ Pull } & \multirow{3}{*}{$\mid v /$} & NS & 138.0189223 & 0.1541950 & 84.3077656 \\
\hline & & P1 & 238.0336763 & 0.0692517 & 75.0521416 \\
\hline & & P2 & 266.7278423 & 0.0919501 & 74.6926275 \\
\hline \multirow{3}{*}{ Best } & \multirow{3}{*}{ /e/ } & NS & 160.0555586 & 0.0839682 & 79.1170257 \\
\hline & & P1 & 187.5225529 & 0.0931519 & 66.9274354 \\
\hline & & P2 & 221.9368334 & 0.1051927 & 70.7746172 \\
\hline \multirow{3}{*}{ About } & \multirow{3}{*}{$/ 2 /$} & NS & 185.5336266 & 0.0593650 & 80.8904046 \\
\hline & & P1 & 218.2287761 & 0.0506802 & 62.6636115 \\
\hline & & $\mathrm{P} 2$ & 241.6990386 & 0.0518594 & 69.7944276 \\
\hline \multirow{3}{*}{ Gas } & \multirow{3}{*}{ /æ/ } & NS & 126.6269865 & 0.2167573 & 79.1256161 \\
\hline & & P1 & 182.7018937 & 0.1030839 & 69.5802465 \\
\hline & & P2 & 240.8210777 & 0.1308163 & 76.4348458 \\
\hline
\end{tabular}




\begin{tabular}{|l|l|l|l|l|l|}
\hline \multirow{3}{*}{ Rush } & \multirow{3}{*}{$/ N /$} & $\mathrm{NS}$ & 179.8797036 & 0.1823582 & 79.1472336 \\
\cline { 3 - 6 } & $\mathrm{P} 1$ & 202.0285300 & 0.1308390 & 71.2826087 \\
\cline { 3 - 6 } & $\mathrm{P} 2$ & 234.3433910 & 0.1381179 & 79.5674276 \\
\hline \multirow{3}{*}{ Gone } & \multirow{3}{*}{$/ \mathrm{D} /$} & $\mathrm{NS}$ & 129.9791799 & 0.1475736 & 83.4747627 \\
\cline { 3 - 6 } & & $\mathrm{P} 1$ & 204.1865726 & 0.1222222 & 70.1553709 \\
\cline { 3 - 6 } & & $\mathrm{P} 2$ & 232.40755504 & 0.1688888 & 75.5524223 \\
\hline
\end{tabular}

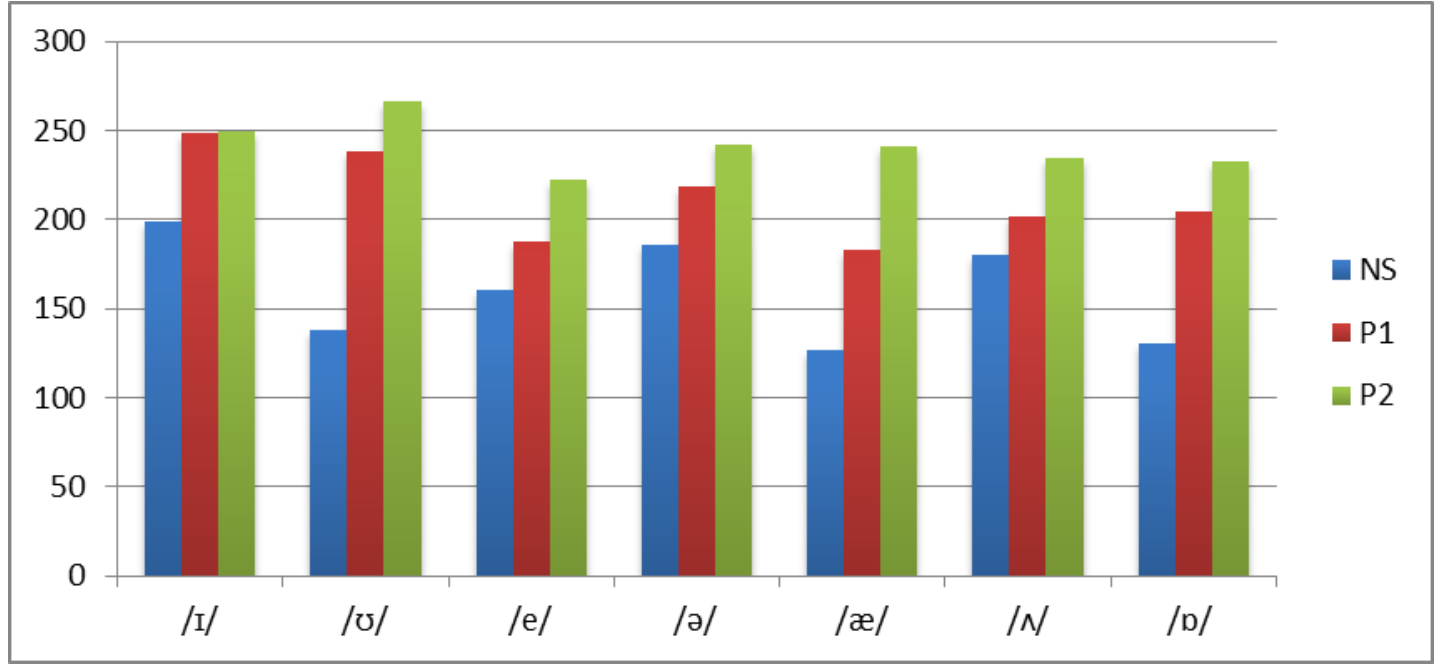

Figure 4.1

Comparative Chart of $/ \mathrm{l} /, / \mho /, / \mathrm{e} /, / \ni /, / æ /, / \Lambda /$, and / $/ \mathrm{p}$ pronounced phonemes Frequency (Pitch)

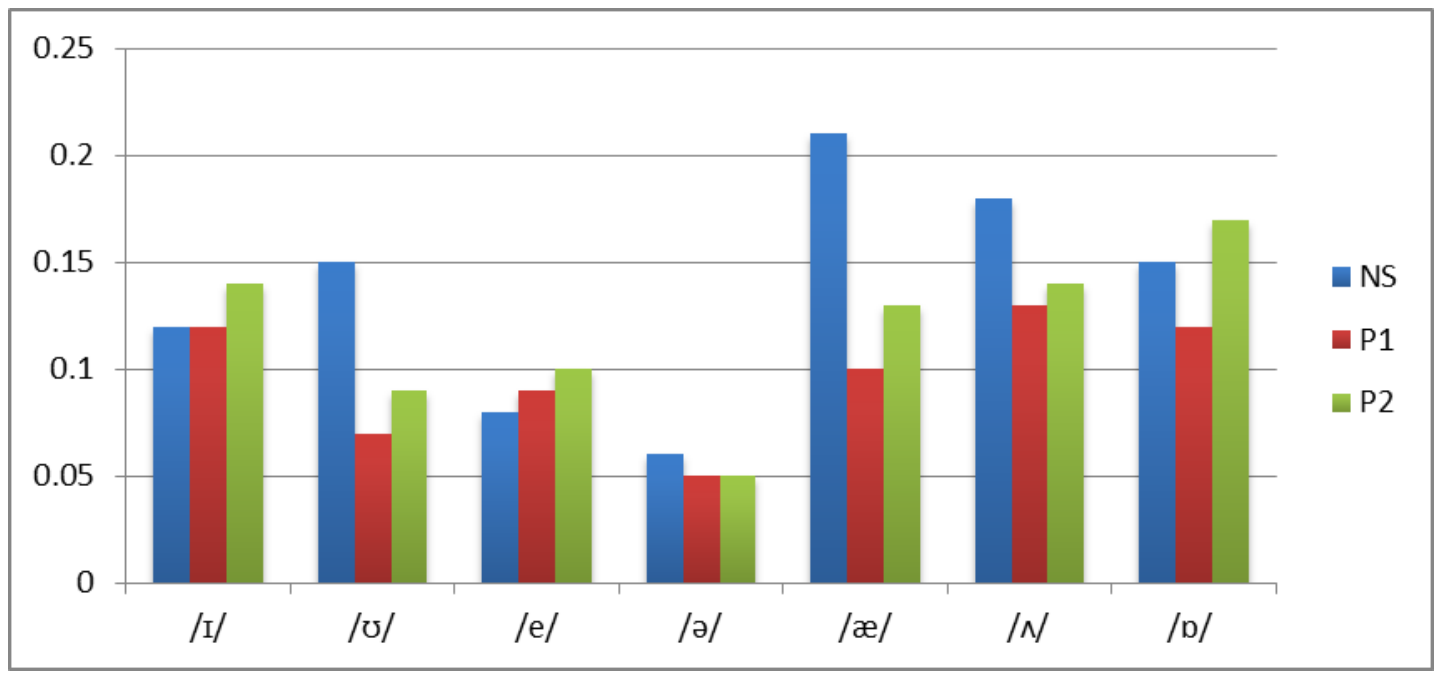

Figure 4.2

Comparative Chart of $/ \mathrm{l} /, / \mathrm{v} /, / \mathrm{e} /, / \mathrm{/} /, / æ /, / \mathrm{N}$, and /o/ pronounced phonemes Quantity (Duration) 
Errors in Pronouncing English Phonemes: A Praat Analysis, Rohani Ganie, Wahyu Maulana, Rahmadsyah Rangkuti

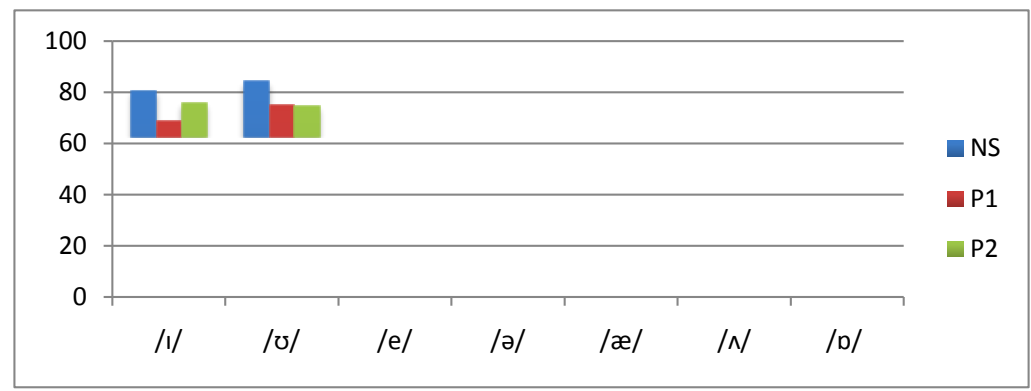

Figure 4.3

Comparative Chart of / //, / /, /e/, /ə/, /æ/, / /, and /o/ pronounced phonemes Intensity (Spectrum)

Table 4.2

Pronounced English Long Vowels

\begin{tabular}{|c|c|c|c|c|c|}
\hline Words & $\begin{array}{l}\text { Focused } \\
\text { Phoneme }\end{array}$ & Speaker & $\begin{array}{c}\text { Frequency } \\
\text { (Pitch) }\end{array}$ & $\begin{array}{c}\text { Quantity } \\
\text { (Duration) }\end{array}$ & $\begin{array}{c}\text { Intensity } \\
\text { (Spectrum) }\end{array}$ \\
\hline \multirow{3}{*}{ Peace } & \multirow{3}{*}{ /i:/ } & NS & 125.1235731 & 0.1687074 & 79.9656268 \\
\hline & & $\mathrm{P} 1$ & 221.7268693 & 0.1945351 & 72.0172887 \\
\hline & & $\mathrm{P} 2$ & 244.5635391 & 0.1686167 & 70.6237845 \\
\hline \multirow{3}{*}{ Loose } & \multirow{3}{*}{ /u:/ } & NS & 134.9429003 & 0.1339682 & 85.5504862 \\
\hline & & $\mathrm{P} 1$ & 217.7961049 & 0.1183900 & 74.2118378 \\
\hline & & $\mathrm{P} 2$ & 247.5602112 & 0.1693197 & 79.1837637 \\
\hline \multirow{3}{*}{ Purse } & \multirow{3}{*}{ /3:/ } & NS & 129.7685407 & 0.1991609 & 79.9246687 \\
\hline & & $\mathrm{P} 1$ & 200.0378063 & 0.1935827 & 71.8306263 \\
\hline & & $\mathrm{P} 2$ & 231.2872364 & 0.1910204 & 76.0491003 \\
\hline \multirow{3}{*}{ Board } & \multirow{3}{*}{ /১:/ } & NS & 135.6118071 & 0.3082993 & 80.5989046 \\
\hline & & $\mathrm{P} 1$ & 192.5357682 & 0.1540589 & 73.1297715 \\
\hline & & $\mathrm{P} 2$ & 227.9374389 & 0.1031519 & 71.8681369 \\
\hline \multirow{3}{*}{ Card } & \multirow{3}{*}{ /a:/ } & NS & 181.3596517 & 0.3423582 & 79.4231112 \\
\hline & & P1 & 196.9557023 & 0.1723809 & 75.5345067 \\
\hline & & $\mathrm{P} 2$ & 228.7128219 & 0.1582086 & 76.3766817 \\
\hline
\end{tabular}

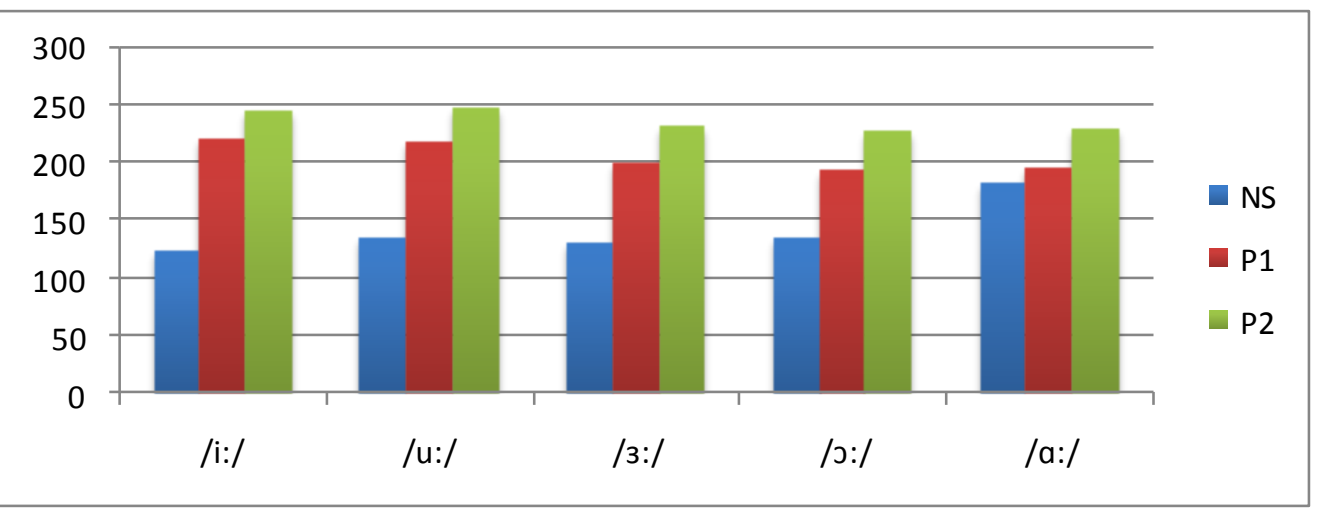

Figure 4.4

Comparative Chart of /i:/, /u:/, /e/, /3:/, / :/, and /a:/ pronounced phonemes Frequency (Pitch). 


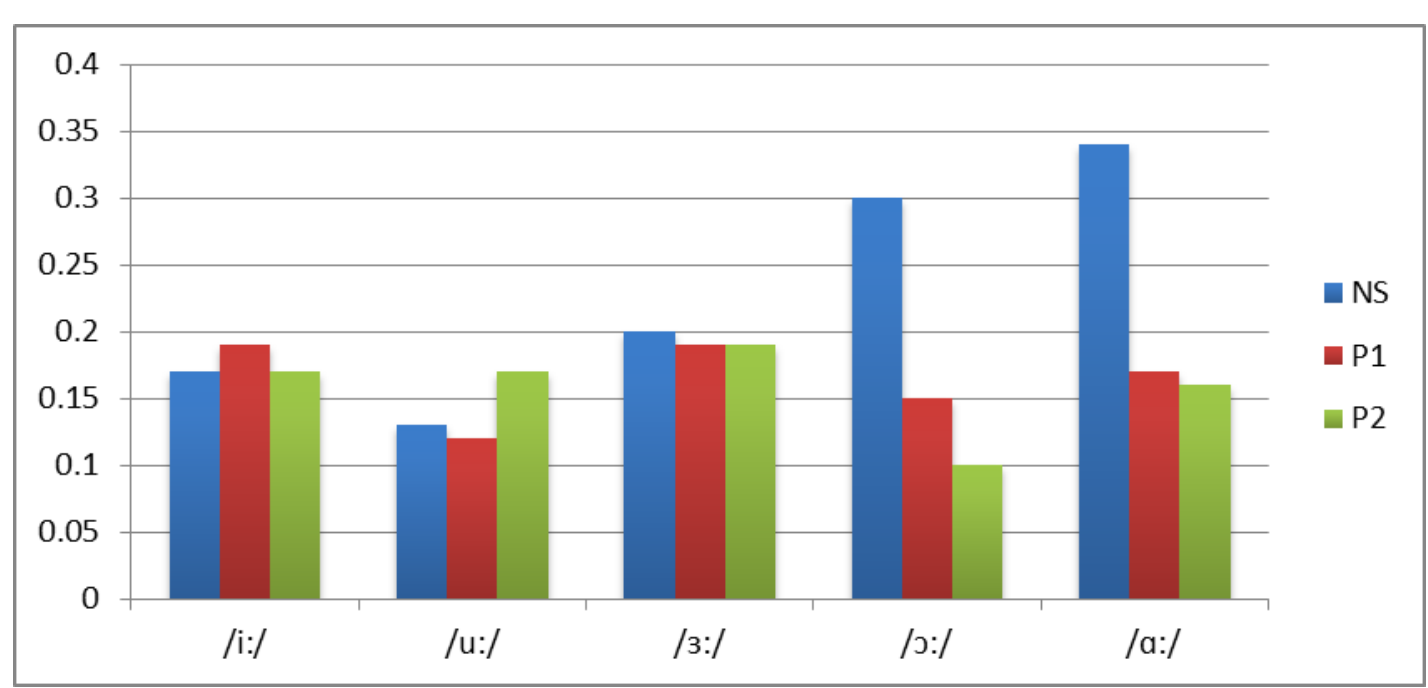

Figure 4.5

Comparative Chart of /i:/, /u:/, /e/, /3:/, / :/, and /a:/ pronunced phonemes Quantity (Duration).

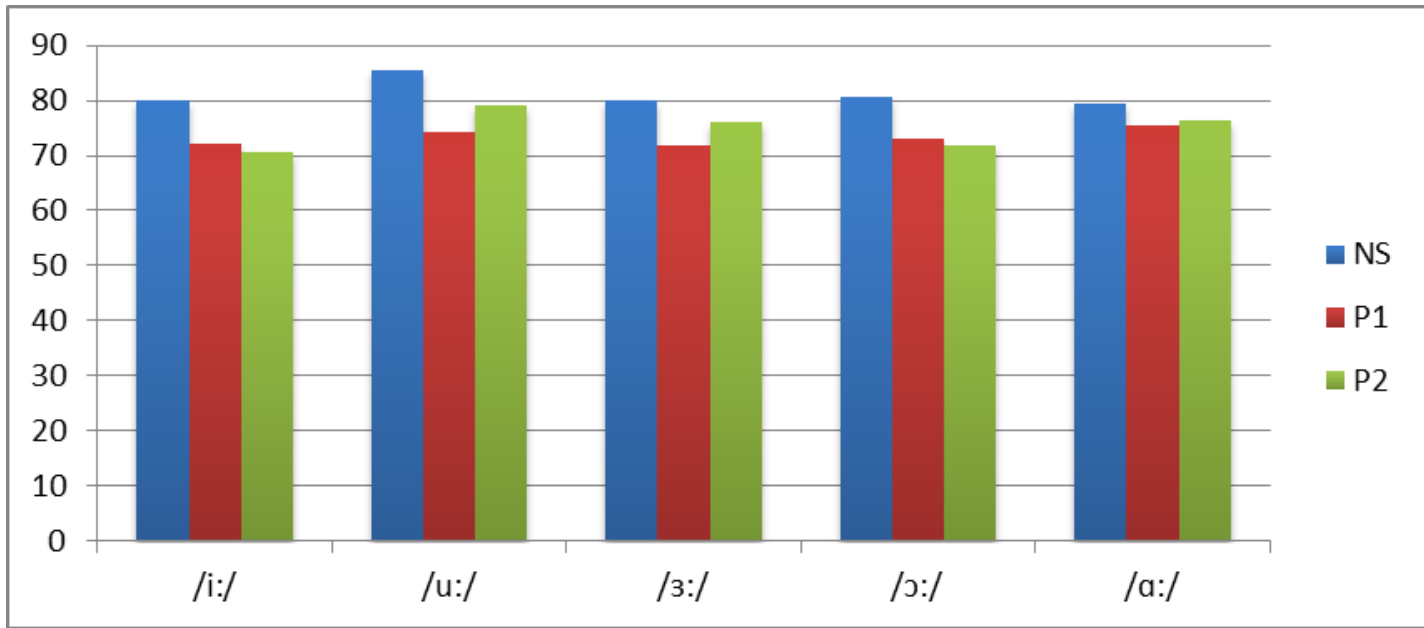

Figure 4.6

Comparative Chart of /i:/, /u:/, /e/, /3:/, / :/, and /a:/ pronounced phonemes Intensity (Spectrum)

Table 4.3 Pronounced English Diphthongs

\begin{tabular}{|c|c|c|c|c|c|}
\hline Words & $\begin{array}{l}\text { Focused } \\
\text { Phoneme }\end{array}$ & Speaker & $\begin{array}{l}\text { Frequency } \\
\text { (Pitch) }\end{array}$ & $\begin{array}{l}\text { Quantity } \\
\text { (Duration) }\end{array}$ & $\begin{array}{l}\text { Intensity } \\
\text { (Spectrum) }\end{array}$ \\
\hline \multirow{3}{*}{ Beard } & \multirow{3}{*}{ /ı/ } & NS & 143.6581892 & 0.2405668 & 80.0332756 \\
\hline & & $\mathrm{P} 1$ & 206.4141379 & 0.1626757 & 74.4964311 \\
\hline & & $\mathrm{P} 2$ & 228.8397334 & 0.1435374 & 75.6047077 \\
\hline \multirow{3}{*}{ Face } & \multirow{3}{*}{ /eı/ } & NS & 139.5285242 & 0.1337868 & 81.5470018 \\
\hline & & P1 & 221.5433358 & 0.1841496 & 68.4517191 \\
\hline & & P2 & 233.0899479 & 0.1870748 & 73.8632397 \\
\hline \multirow{3}{*}{ Tour } & \multirow{3}{*}{ /ఠə/ } & NS & 175.0336835 & 0.2383446 & 81.4429501 \\
\hline & & P1 & 228.6960855 & 0.2069614 & 71.5874041 \\
\hline & & $\mathrm{P} 2$ & 235.2231062 & 0.2204308 & 72.9050424 \\
\hline
\end{tabular}


Errors in Pronouncing English Phonemes: A Praat Analysis, Rohani Ganie, Wahyu Maulana, Rahmadsyah Rangkuti

\begin{tabular}{|c|c|c|c|c|c|}
\hline \multirow{3}{*}{ Voice } & \multirow{3}{*}{ /וכ// } & NS & 130.9559368 & 0.2116780 & 81.2437156 \\
\hline & & P1 & 206.9275107 & 0.1972108 & 70.9962223 \\
\hline & & $\mathrm{P} 2$ & 242.7422494 & 0.2041269 & 75.1438418 \\
\hline \multirow{3}{*}{ Home } & \multirow{3}{*}{ /əఠ/ } & NS & 146.2544160 & 0.1431292 & 83.7180472 \\
\hline & & P1 & 216.6685880 & 0.1327664 & 74.8978918 \\
\hline & & $\mathrm{P} 2$ & 247.3277399 & 0.1309977 & 76.4468057 \\
\hline \multirow{3}{*}{ Scarce } & \multirow{3}{*}{ /еә/ } & NS & 128.6710744 & 0.2606575 & 79.2718072 \\
\hline & & P1 & 204.1208010 & 0.1977324 & 67.3742116 \\
\hline & & $\mathrm{P} 2$ & 258.7629195 & 0.1609750 & 76.1914212 \\
\hline \multirow{3}{*}{ Time } & \multirow{3}{*}{ /aı/ } & NS & 135.3016691 & 0.2175510 & 83.4063538 \\
\hline & & P1 & 213.2227340 & 0.2238548 & 72.6770321 \\
\hline & & $\mathrm{P} 2$ & 231.2182619 & 0.2087528 & 73.0775257 \\
\hline \multirow{3}{*}{ Loud } & \multirow{3}{*}{ /av/ } & NS & 123.9513831 & 0.2655782 & 77.4273393 \\
\hline & & P1 & 188.1956915 & 0.1399319 & 70.4168189 \\
\hline & & $\mathrm{P} 2$ & 220.4925342 & 0.2210430 & 75.8466901 \\
\hline
\end{tabular}

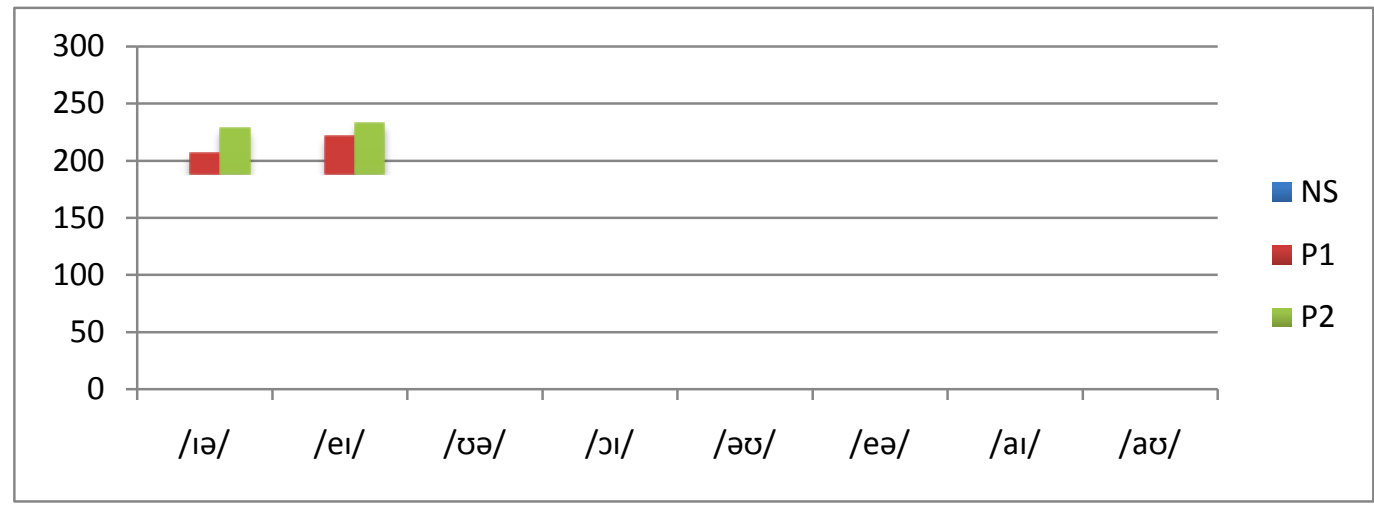

Figure 4.7

Comparative Chart of /ı/, /eı/, /və/, /כı/, /əv/, /eə/, /aı/, and /av/ pronounced phonemes Frequency (Pitch).

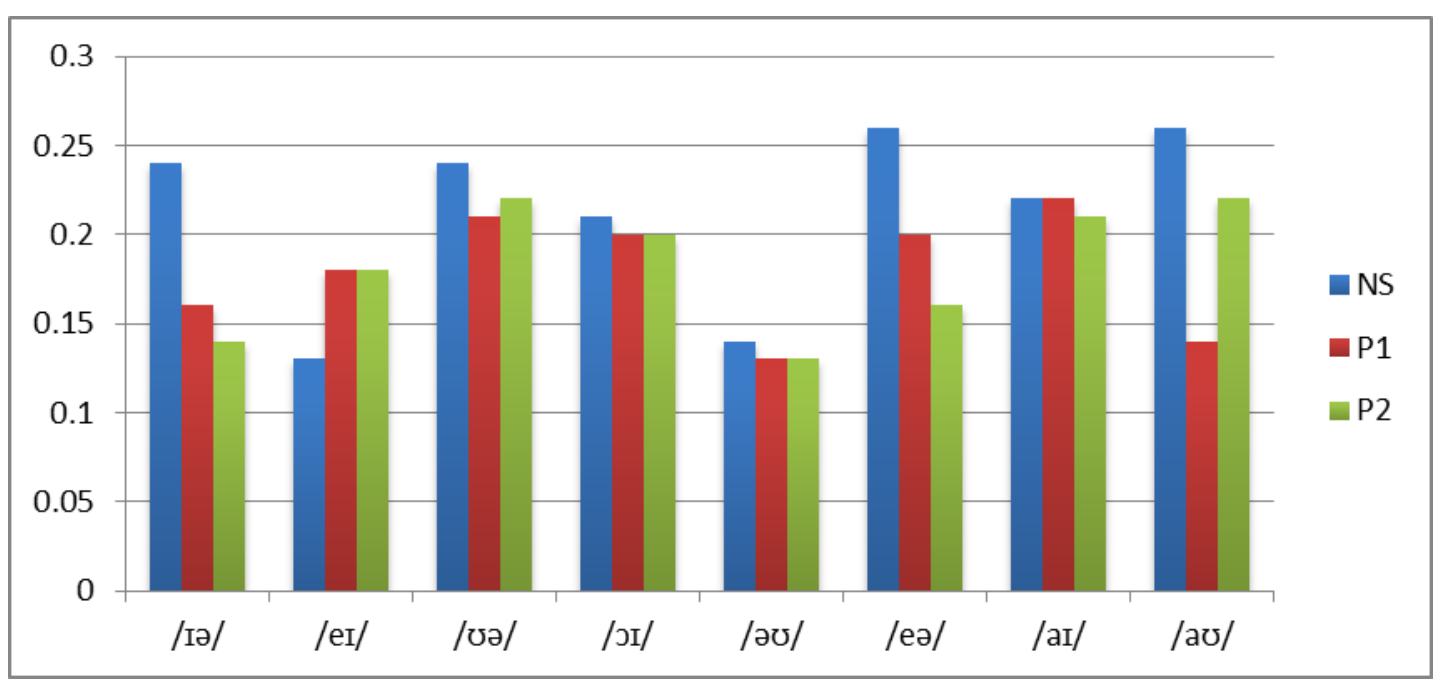

Figure 4.8

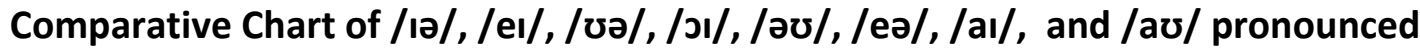
phonemes Quantity (Duration) 


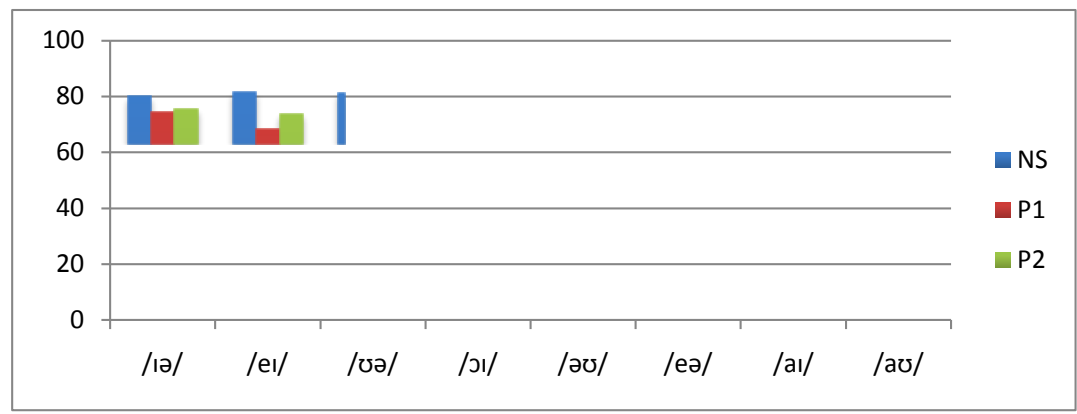

Figure 4.9

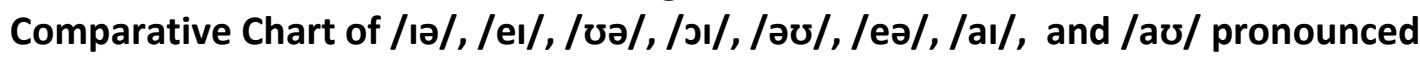
phonemes Intensity (Spectrum)

Table 4.10 Pronounced English Triphthongs

\begin{tabular}{|c|c|c|c|c|c|}
\hline Words & $\begin{array}{l}\text { Focused } \\
\text { Phoneme }\end{array}$ & Speaker & $\begin{array}{l}\text { Frequency } \\
\text { (Pitch) }\end{array}$ & $\begin{array}{l}\text { Quantity } \\
\text { (Duration) }\end{array}$ & $\begin{array}{l}\text { Intensity } \\
\text { (Spectrum) }\end{array}$ \\
\hline \multirow{3}{*}{ Layer } & \multirow{3}{*}{ /еıә/ } & NS & 177.9743725 & 0.3074376 & 79.4330650 \\
\hline & & P1 & 200.8384674 & 0.3468934 & 68.3665787 \\
\hline & & P2 & 244.2132866 & 0.3352380 & 75.3942525 \\
\hline \multirow{3}{*}{ Liar } & \multirow{3}{*}{ /аıә/ } & NS & 180.7913466 & 0.2501360 & 78.5085299 \\
\hline & & P1 & 200.4783038 & 0.3879365 & 69.1844105 \\
\hline & & $\mathrm{P} 2$ & 231.6686971 & 0.3236507 & 76.0918328 \\
\hline \multirow{3}{*}{ Royal } & \multirow{3}{*}{ /دા/ } & NS & 183.2083471 & 0.3241269 & 81.7665817 \\
\hline & & $\mathrm{P} 1$ & 200.3163959 & 0.2744217 & 68.3683304 \\
\hline & & $\mathrm{P} 2$ & 249.5102718 & 0.3158276 & 77.9146459 \\
\hline \multirow{3}{*}{ Lower } & \multirow{3}{*}{ /əఠə/ } & NS & 170.3535340 & 0.2538775 & 78.0752508 \\
\hline & & $\mathrm{P} 1$ & 215.7125501 & 0.2428571 & 71.4687629 \\
\hline & & P2 & 238.2985780 & 0.3714058 & 76.4851962 \\
\hline \multirow{3}{*}{ Power } & \multirow{3}{*}{ /аचə/ } & NS & 173.8672834 & 0.3476190 & 80.2162826 \\
\hline & & P1 & 223.9827599 & 0.2601587 & 70.4867897 \\
\hline & & P2 & 237.0149105 & 0.2808163 & 76.2742223 \\
\hline
\end{tabular}

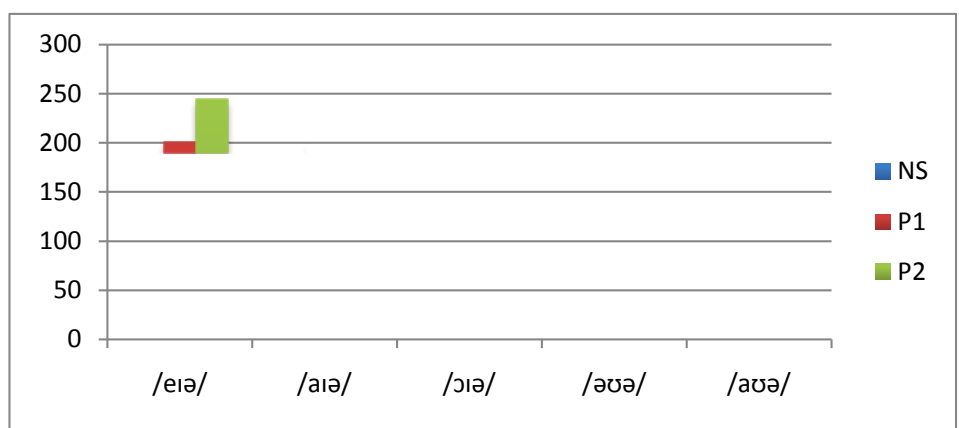

Figure 4.10

Comparative Chart of /eıә/, /аı/, /Јә/, /әбә/, and /avə/pronounced phonemes Frequency (Pitch). 
Errors in Pronouncing English Phonemes: A Praat Analysis, Rohani Ganie, Wahyu Maulana, Rahmadsyah Rangkuti

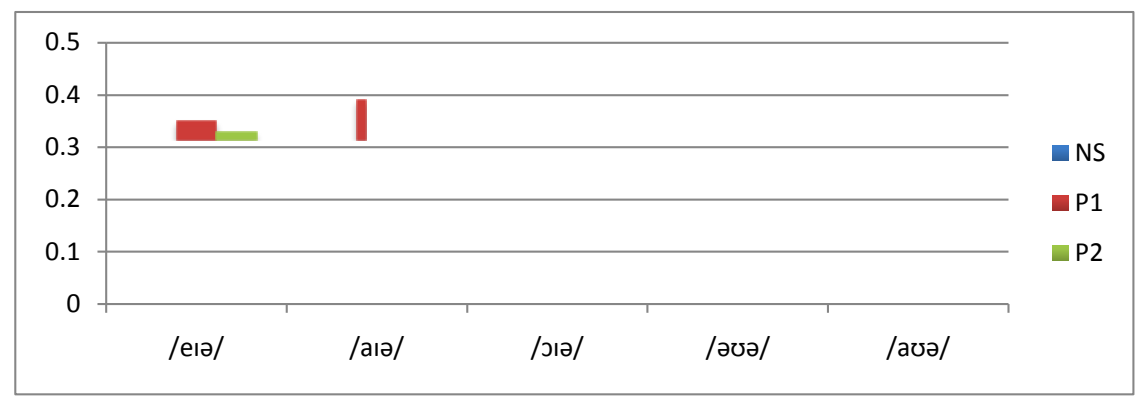

Figure 4.11

Comparative Chart of /eı/, /аıә/, /Јә/, /әәә/, and /avə/ pronounced phonemes Quantity (Duration).

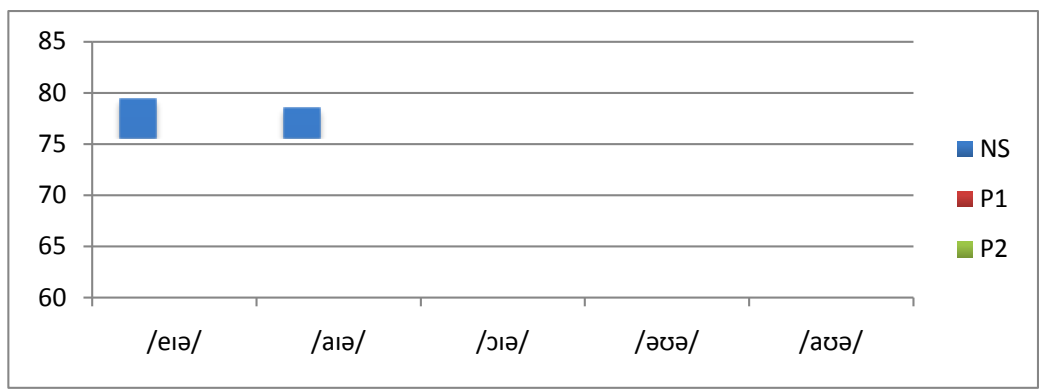

Figure 4.12

Comparative Chart of /eı/, /aı/, /כә/, /әәә/, and /avə/pronounced phonemes Intensity (Spectrum)

Table 4.5

Pronounced English Voiced Consonants

\begin{tabular}{|c|c|c|c|c|c|}
\hline Words & $\begin{array}{l}\text { Focused } \\
\text { Phoneme }\end{array}$ & Speaker & $\begin{array}{c}\text { Frequency } \\
\text { (Pitch) }\end{array}$ & $\begin{array}{l}\text { Quantity } \\
\text { (Duration) }\end{array}$ & $\begin{array}{l}\text { Intensity } \\
\text { (Spectrum) }\end{array}$ \\
\hline \multirow{3}{*}{ Ball } & \multirow{3}{*}{ /b/ } & NS & 142.0190310 & 0.0384126 & 80.4902777 \\
\hline & & P1 & 197.0051919 & 0.1275056 & 63.2785110 \\
\hline & & P2 & 224.9774747 & 0.1160090 & 63.3221333 \\
\hline \multirow{3}{*}{ Dog } & \multirow{3}{*}{ /d/ } & NS & 548.6544503 & 0.0194331 & 66.1037454 \\
\hline & & P1 & 188.9661797 & 0.1294104 & 60.7780465 \\
\hline & & P2 & 218.5419691 & 0.0886621 & 60.4339423 \\
\hline \multirow{3}{*}{ Joke } & \multirow{3}{*}{$/ d z /$} & NS & 317.7665421 & 0.0649433 & 67.6260170 \\
\hline & & P1 & 223.2543332 & 0.0897732 & 62.8682940 \\
\hline & & $\mathrm{P} 2$ & 85.8210937 & 0.1092517 & 62.4381700 \\
\hline \multirow{3}{*}{ Good } & \multirow{3}{*}{ /g/ } & NS & 386.6681321 & 0.0261451 & 56.9652317 \\
\hline & & P1 & 183.3045308 & 0.1278684 & 61.1951245 \\
\hline & & P2 & 117.2616705 & 0.0955328 & 57.3303761 \\
\hline \multirow{2}{*}{ Van } & \multirow{2}{*}{$/ \mathrm{v} /$} & NS & 816.8996666 & 0.0580725 & 56.6712832 \\
\hline & & P1 & 106.2296262 & 0.0703174 & 49.7500213 \\
\hline
\end{tabular}


Language Literacy: Journal of Linguistics, Literature and Language Teaching

Volume 3, Number 1, pp: 49-63, June 2019

e-ISSN: 2580-9962 | p-ISSN: 2580-8672

DOI: $10.30743 / /$ II.v3i1.1216

\begin{tabular}{|c|c|c|c|c|c|}
\hline & & $\mathrm{P} 2$ & 82.3251742 & 0.1008843 & 63.2379565 \\
\hline \multirow{3}{*}{ Father } & \multirow{3}{*}{ /ð/ } & NS & 163.6029011 & 0.0914739 & 55.1591239 \\
\hline & & $\mathrm{P} 1$ & 197.1575835 & 0.1187528 & 55.8848908 \\
\hline & & $\mathrm{P} 2$ & 258.6481177 & 0.0816326 & 59.1931969 \\
\hline \multirow{3}{*}{ Zip } & \multirow{3}{*}{ /z/ } & NS & 142.3905624 & 0.1070521 & 77.5744600 \\
\hline & & P1 & 184.3538143 & 0.1821088 & 62.6994806 \\
\hline & & $\mathrm{P} 2$ & 124.2957748 & 0.1187528 & 67.9363752 \\
\hline \multirow{3}{*}{ Measure } & \multirow{3}{*}{$/ 3 /$} & NS & 168.4574086 & 0.1010430 & 70.2525075 \\
\hline & & P1 & 195.1559060 & 0.1349659 & 62.8181926 \\
\hline & & $\mathrm{P} 2$ & 127.0967443 & 0.0898866 & 69.0725068 \\
\hline \multirow{3}{*}{ Mouth } & \multirow{3}{*}{$/ \mathrm{m} /$} & NS & 144.9547490 & 0.0706122 & 75.8811390 \\
\hline & & $\mathrm{P} 1$ & 208.7318441 & 0.0809750 & 68.6478201 \\
\hline & & $\mathrm{P} 2$ & 252.2413731 & 0.0831972 & 66.8093397 \\
\hline \multirow{3}{*}{ Nothing } & \multirow{3}{*}{$/ \mathrm{n} /$} & NS & 141.2716999 & 0.0646031 & 73.0558055 \\
\hline & & P1 & 105.3213255 & 0.1224943 & 65.7888466 \\
\hline & & $\mathrm{P} 2$ & 261.1503793 & 0.0851247 & 70.2147828 \\
\hline \multirow{3}{*}{ Sing } & \multirow{3}{*}{$/ \mathrm{h} /$} & NS & 122.0032681 & 0.2002040 & 71.6309167 \\
\hline & & P1 & 177.3683848 & 0.3697278 & 61.0053751 \\
\hline & & $\mathrm{P} 2$ & 106.4999278 & 0.1691156 & 66.6099851 \\
\hline \multirow{3}{*}{ Love } & \multirow{3}{*}{ /I/ } & NS & 143.9455229 & 0.0626077 & 79.1297765 \\
\hline & & $\mathrm{P} 1$ & 208.8140675 & 0.0884580 & 59.1844073 \\
\hline & & $\mathrm{P} 2$ & 240.6561522 & 0.0912244 & 66.1799387 \\
\hline \multirow{3}{*}{ Red } & \multirow{3}{*}{$/ r /$} & NS & 95.9571633 & 0.1173242 & 76.4888507 \\
\hline & & $\mathrm{P} 1$ & 185.7829578 & 0.1137414 & 61.8773646 \\
\hline & & $\mathrm{P} 2$ & 123.5727384 & 0.0829931 & 65.2957593 \\
\hline \multirow{3}{*}{ Witch } & \multirow{3}{*}{ /w/ } & NS & 150.3407784 & 0.0477097 & 83.0318542 \\
\hline & & $\mathrm{P} 1$ & 206.8665780 & 0.1190702 & 65.5268053 \\
\hline & & $\mathrm{P} 2$ & 249.2914686 & 0.0680952 & 64.9178881 \\
\hline \multirow{3}{*}{ Yes } & \multirow{3}{*}{ /j/ } & NS & 193.9523486 & 0.1235827 & 78.7804051 \\
\hline & & P1 & 210.4515144 & 0.0675736 & 64.7903915 \\
\hline & & $\mathrm{P} 2$ & 247.1797304 & 0.1100453 & 67.6117301 \\
\hline
\end{tabular}

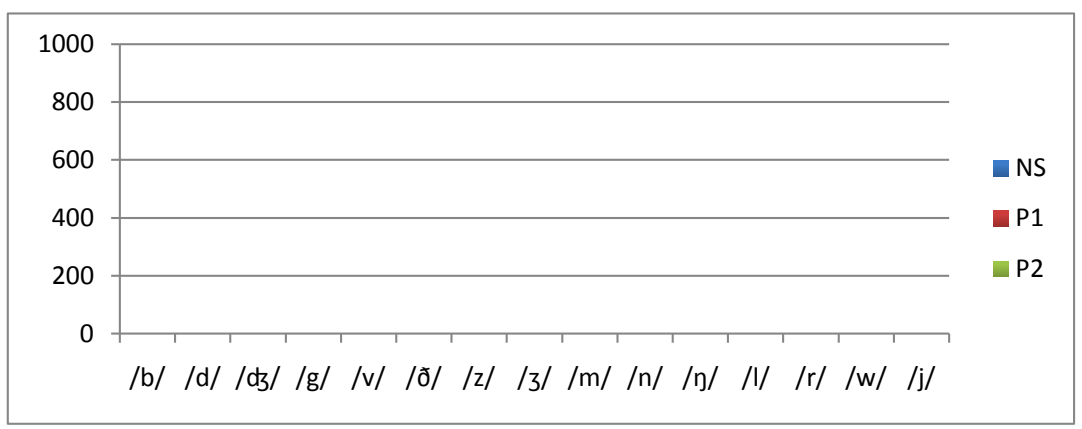

Figure 4.13

Comparative Chart of /b/, /d/, /dz/, /g/, /v/, /ð/,/z/,/3/, /m/, /n/, /y/, /l/, /r/, /w/, and $/ \mathrm{j} /$ pronounced phonemes Frequency (Pitch) 
Errors in Pronouncing English Phonemes: A Praat Analysis, Rohani Ganie, Wahyu Maulana, Rahmadsyah Rangkuti

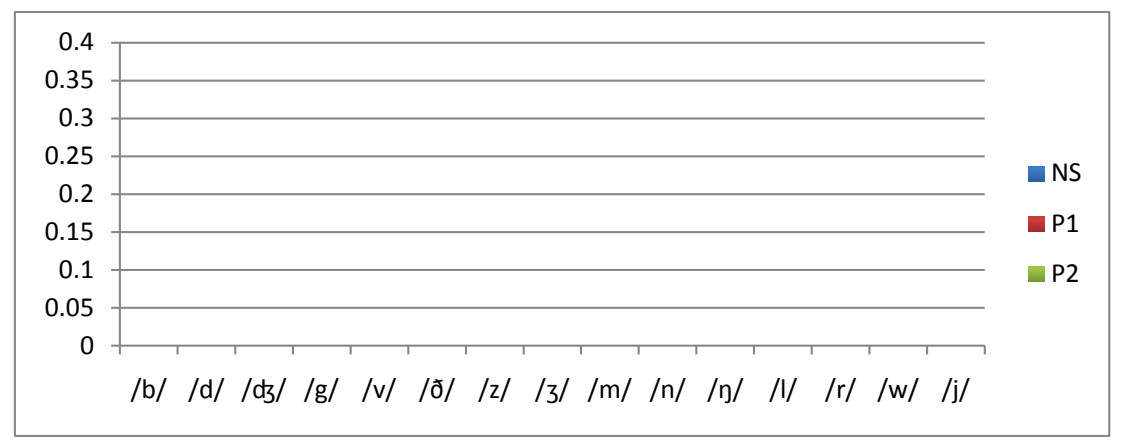

Figure 4.14

Comparative Chart of /b/, /d/, /ds/, /g/, /v/, /ठ/, /z/, /3/, /m/, /n/, /y/, /l/, /r/, /w/, and /j/ pronunced phonemes Quantity (Duration)

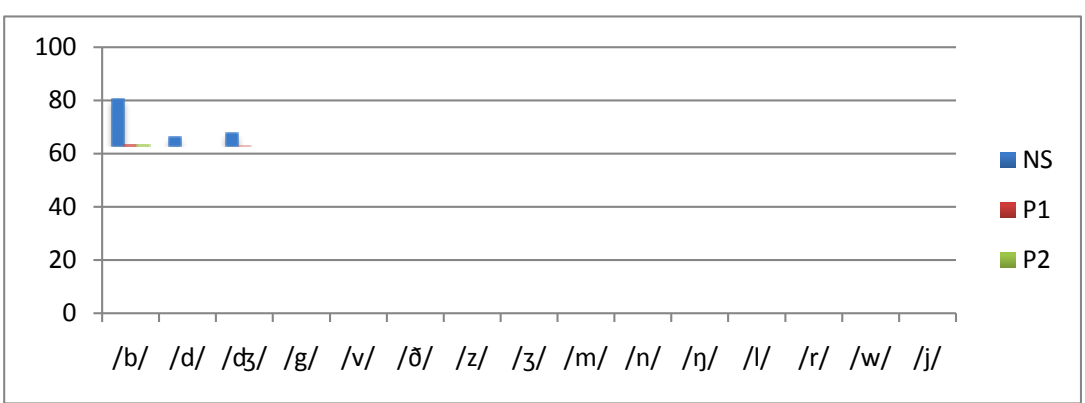

Figure 4.15

Comparative Chart of /b/, /d/, /dz/, /g/, /v/, / /, /z/, /3/, /m/, /n/, /g/, /l/, /r/, /w/, and /j/ pronounced phonemes Intensity (Spectrum)

4.6 Pronounced English Voiceless Consonants

\begin{tabular}{|c|c|c|c|c|c|}
\hline Words & $\begin{array}{l}\text { Focused } \\
\text { Phoneme }\end{array}$ & Speaker & $\begin{array}{l}\text { Frequency } \\
\text { (Pitch) }\end{array}$ & $\begin{array}{l}\text { Quantity } \\
\text { (Duration) }\end{array}$ & $\begin{array}{l}\text { Intensity } \\
\text { (Spectrum) }\end{array}$ \\
\hline \multirow{3}{*}{ Pea } & \multirow{3}{*}{$/ \mathrm{p} /$} & NS & 438.0859259 & 0.0478684 & 59.0593833 \\
\hline & & $\mathrm{P} 1$ & 209.5659509 & 0.0425396 & 68.9877762 \\
\hline & & $\mathrm{P} 2$ & 280.9217014 & 0.0480952 & 66.7773536 \\
\hline \multirow{3}{*}{ Tea } & \multirow{3}{*}{$/ \mathrm{t} /$} & NS & 454.4883474 & 0.0762131 & 69.7431705 \\
\hline & & $\mathrm{P} 1$ & 229.1721206 & 0.0419274 & 61.6244638 \\
\hline & & $\mathrm{P} 2$ & 183.8807165 & 0.0620861 & 57.9109610 \\
\hline \multirow{3}{*}{ Nature } & \multirow{3}{*}{$/ \mathrm{t} /$} & NS & 219.0441825 & 0.1128798 & 71.3044893 \\
\hline & & $\mathrm{P} 1$ & 221.9069718 & 0.1265986 & 66.9267995 \\
\hline & & $\mathrm{P} 2$ & 452.8307220 & 0.0665759 & 68.2111688 \\
\hline \multirow{3}{*}{ Coffee } & \multirow{3}{*}{$/ \mathrm{k} /$} & NS & 421.1288441 & 0.0600453 & 57.6402177 \\
\hline & & P1 & 288.8670563 & 0.0398412 & 60.2988311 \\
\hline & & $\mathrm{P} 2$ & 501.8464336 & 0.0494104 & 60.0795973 \\
\hline Fan & $/ f /$ & NS & 312.7676220 & 0.0894784 & 56.8550984 \\
\hline
\end{tabular}




\begin{tabular}{|c|c|c|c|c|c|}
\hline & & P1 & 197.1575835 & 0.1187528 & 55.8848908 \\
\hline & & $\mathrm{P} 2$ & 421.0557913 & 0.1376190 & 71.1938704 \\
\hline \multirow{3}{*}{ Thumb } & \multirow{3}{*}{$/ \theta /$} & NS & 482.3518872 & 0.1365306 & 58.0026577 \\
\hline & & P1 & 2160.4670428 & 0.1728571 & 55.2341428 \\
\hline & & $\mathrm{P} 2$ & 121.7426010 & 0.0809297 & 56.3006397 \\
\hline \multirow{3}{*}{ Facing } & \multirow{3}{*}{ /s/ } & NS & 233.0346168 & 0.1449659 & 75.8341821 \\
\hline & & $\mathrm{P} 1$ & 239.3403475 & 0.1840589 & 71.9209567 \\
\hline & & $\mathrm{P} 2$ & 96.4129415 & 0.1400226 & 72.4158887 \\
\hline \multirow{3}{*}{ Ship } & \multirow{3}{*}{$/ \delta /$} & NS & 316.2145589 & 0.1596371 & 70.9223151 \\
\hline & & $\mathrm{P} 1$ & 491.5341962 & 0.2515873 & 74.8917669 \\
\hline & & $\mathrm{P} 2$ & 178.1640505 & 0.1481859 & 68.6016945 \\
\hline \multirow{3}{*}{ Ahead } & \multirow{3}{*}{ /h/ } & NS & 339.4688525 & 0.1340136 & 64.8746212 \\
\hline & & $\mathrm{P} 1$ & 126.7776866 & 0.1360770 & 61.4045972 \\
\hline & & $\mathrm{P} 2$ & 114.9191008 & 0.1407482 & 64.6725592 \\
\hline
\end{tabular}

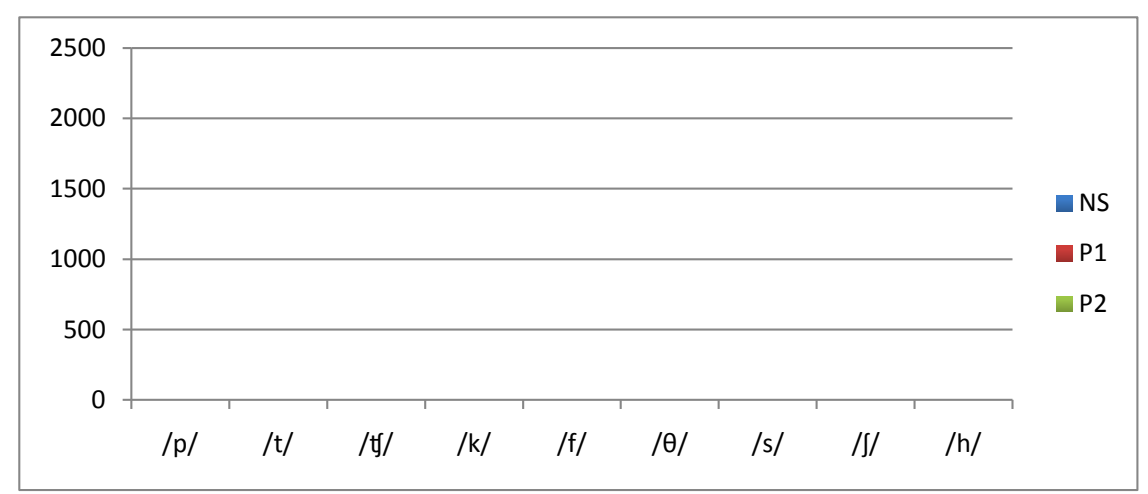

Figure 4.16

Comparative Chart of $/ \mathrm{p} /, / \mathrm{t} /, / \mathrm{t} /, / \mathrm{k} /, / \mathrm{f} /, / \theta /, / \mathrm{s} /, / \mathrm{s} /$, and $/ \mathrm{h} /$ pronounced phonemes Frequency (Pitch)

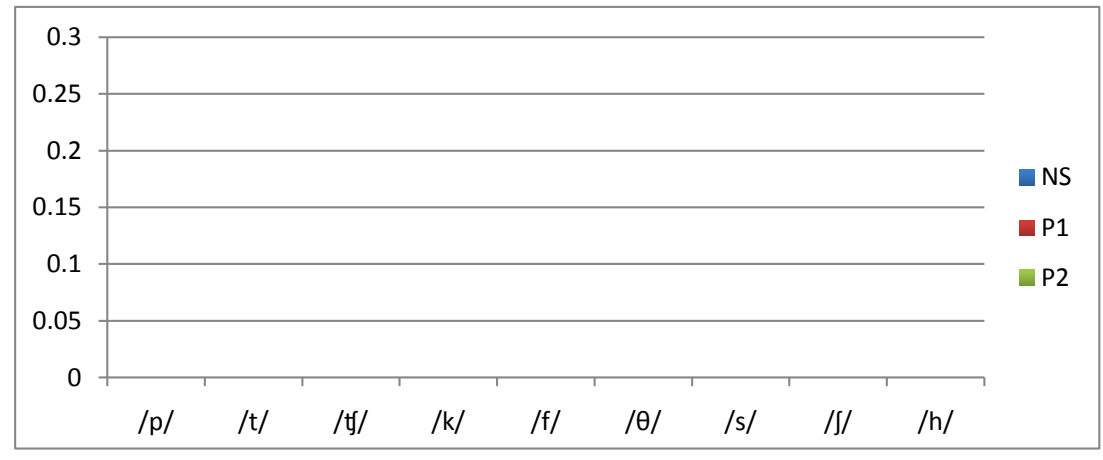

Figure 4.17

Comparative Chart of $/ \mathrm{p} /, / \mathrm{t} /, / \mathrm{t} /, / \mathrm{k} /, / \mathrm{f} /, / \theta /, / \mathrm{s} /, / \mathrm{s} /$, and $/ \mathrm{h} /$ pronounced phonemes Quantity (Duration) 
Errors in Pronouncing English Phonemes: A Praat Analysis, Rohani Ganie, Wahyu Maulana, Rahmadsyah Rangkuti

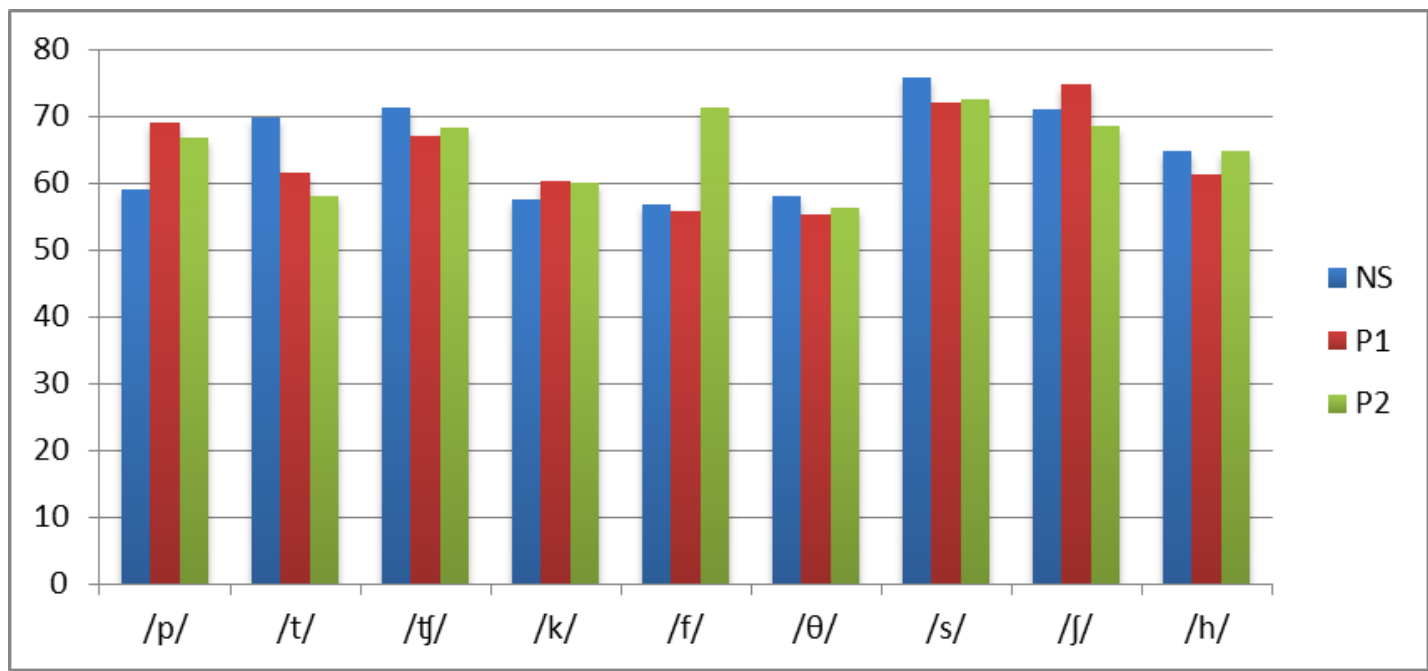

Figure 4.18

\section{Comparative Chart of $/ \mathrm{p} /, / \mathrm{t} /, / \mathrm{t} /, / \mathrm{k} /, / \mathrm{f} /, / \theta /, / \mathrm{s} /, / \mathrm{s} /$, and $/ \mathrm{h} /$ pronounced phonemes Intensity (Spectrum)}

To compare the participants' pronunciation value in percentage, the Native Speaker's pronunciation is considered $100 \%$ correct. This value is not a maximal value as the value of the participants' acoustic terms varies in number. It could be lower than the native speaker or higher but this value is the standard. If the participants' pronunciation value is less than $100 \%$, it is considered as low and vice versa.

To judge the participants' errors, the researcher took each value to be compared with the native speaker's value in the range between the participants' pronunciation and the native speaker's pronunciation. If the range of the participants' pronunciation is wider, it means that the errors are higher. The errors are sorted from high to low.

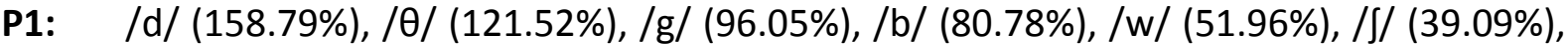
/g/ (38.51\%), /t/ (37.07\%), /v/ (27.51\%), /al/ (27.06\%), /m/ (26.25\%), /z/ (24.65\%), /l/ (23.28\%), /r/ (22.05\%), /n/ (21.54\%), /n/ (20.04\%), /p/ (18.57\%), /ð/ (18.38\%), /aı/ (18.34\%), /j/ (16.97\%), /h/ (16.37\%), /a:/ (15.43\%), /aı/ (14.91\%), /ı/ (13.55\%), /u:/ (13.48\%), /з:/ (13.01\%), /3/ (11.75\%), /әб/ (10.16\%), / / (8.47\%), /i:/ (8.01\%), /دә/ (7.56\%), /æ/ (7.45\%), /ə/ (7.15\%), /o/ (7.04\%), /eə/ (6.85\%), /s/ (6.33\%), / :/ (6.31\%), /e/ (4.75\%), /әбә/ (4.73\%), /t/ (4.45\%), /d3/ (4.40\%), /v/ (4.05\%), /eı/ (3.94\%), /I/ (3.31\%), /aбə/ (3.01\%), /və/ (2.02\%), /f/ (1.78\%), /ı/ (1.15\%), /av/ (1.12\%).

P2: $\quad$ /d/ (93.75\%), /b/ (79.03\%), /w/ (44.27\%), /g/ (43.66\%), / / / (40.18\%), /f/ (38.47), /n/ (38.10), /u:/ (35.59), /s/ (35.33), /el/ (34.96), /t/ (33.83), /l/ (33.61), /əซə/ (28.28), /i:/ (27.93), /p/ (27.55), /m/ (25.47), /з/ (24.37), /3:/ (22.79), /t/ (22.01), /av/ (20.15), /h/ (19.59), /eə/ (19.58), /ð/ (18.09), /aı/ (17.98), /əб/ (17.76), /J/ (17.73), /e/ (17.70), /aı/ (17.69), /ひ/ (13.66), /eı/ (12.86), /3/ (12.08), /ı/ (12.01), /y/ (11.57), /a:/ (10.22), /دә/ (9.45), /p/ (7.61), /r/ (6.40), /z/ (5.35), /æ/ (5.29), /ひə/ (5.19), /ı/ (4.03), /v/ (3.89), /aøə/ (3.79), /د:/ (3.47), / / (2.86), /n/ (2.24), /j/ (1.64), /d3/ (0.89), /ə/ (0.04).

\section{Conclusion}

The conclusion comes from the data analysis and findings in the previous chapter. The first purpose is to show the incorrect participants' pronunciation of phonemes in graphic charts and percentage tables. The data for graphic charts and percentage tables come from Praat software. This software synthesizes the students' pronunciation in sound wave and it 
comes along with the value. This value is then extracted in charts and compared with the native speaker's pronunciation value. The result shows that no participants pronounce English phonemes correctly or $0 \%$ range value. Finally, the participants' pronunciation is analyzed from the value of the pitch, duration, and spectrum by using Praat software. The value of each phoneme is then summed up and divided by three to find the average value. The average value of correct pronunciation of English phonemes is $100 \%$.

It was also found that Praat software is very helpful in doing this research, but the researcher also had hard times to learn and develop knowledge of how to run this software at the first time. So the researcher suggests that students in the department of English need to learn how to use this software to help them do significant phonetic research in future.

\section{References}

Katamba, Francis. (1996). An introduction to phonology. Eighth impression. London and New York: Longman.

Kurniawan, Dedi. (2016). The error analysis of the pronunciation of dental fricative consonants $(/ \theta /, / \circlearrowright /)$ by students of English education study program Faculty of Teacher Training and Education Sriwijaya University. Palembang: Journal of English Literacy Education.

Moleong, Lexy J. (2000). Metodologi penelitian kualitatif. Bandung: Remaja Rosda Karya.

Nilawati, Nilawati. (2008). The fossilized phones errors of the English Department students of Andalas University: An inter language study. Padang: Fakultas Sastra Universitas Andalas.

Ogden, Richard. (2009). An introduction to English phonetics. Edinburgh: Edinburgh University Press.

Perpustakaan UGM, i-lib. (2003). Interferensi fonologi Bahasa Indonesia dalam Bahasa Perancis: Phonologic Interference of Indonesian in French. Yogyakarta: Jurnal i-lib UGM.

Roach, Peter. (2008). English phonetics and phonology. Cambridge: Cambridge University Press.

University of Cambridge. (2008). Cambridge advanced learner's dictionary. Third edition. Cambridge: Cambridge University Press.

Wikipedia. (2019). (https://en.wikipedia.org/wiki/ Praat). Accessed on March 20 ${ }^{\text {th }}, 2019$. 